\title{
Transient Radiographic Early Enhancement after Radiotherapy for Meningioma
}

\author{
Abhirami Hallock, Glenn Bauman
}

Can. J. Neurol. Sci. 2012; 39: 99-101

The role of adjuvant and primary radiotherapy for meningioma has been based largely on individual institutional retrospective experiences and ongoing prospective trials seek to better define the role of radiotherapy for these tumors. ${ }^{1}$ Typically, meningiomas exhibit stable imaging appearances post radiation with new or worsening enhancement usually indicating tumor progression or recurrence. Transient radiographic changes as a consequence of treatment effect secondary to fractionated radiotherapy has been described for malignant glioma. ${ }^{2}$ However, to the best of our knowledge, this phenomenon has not been described for meningiomas. We report a case of a meningioma treated with primary radiation that demonstrated reversible enhancement on post radiation treatment magnetic resonance imaging (MRI), distinct from a typical pattern of delayed radiation necrosis or tumor progression after stereotactic fractionated radiotherapy.

A 64-year-old female presented with gradual worsening vision of her left eye. Physical examination showed ptosis, near complete vision loss with relative afferent papillary defect of the left eye. Magnetic resonance imaging showed a $5 \mathrm{~cm}$ left cavernous sinus enhancing mass consistent with a meningioma (Figure 1a), with complete encasement of the left optic nerve, carotids and extension into the anterior middle fossa. The well circumscribed, enhancing nature of the lesion, absence of edema or brain invasion and long clinical course was suggestive of a World Health Organization Grade I meningioma. ${ }^{1}$ Management options (surgery, surveillance, radiotherapy) were discussed with the patient who chose to undergo a radical course of radiation with the goal of preservation of her remaining vision. Size, and proximity to critical structures did not allow radiosurgery and she was treated with intensity modulated fractionated stereotactic radiotherapy with a prescribed dose of 54 Gy in 30 fractions. A thermoplastic shell was used for immobilization and daily orthogonal two dimensional kilovoltage imaging was used for daily localization and positioning correction. Computed tomogram (CT) and MRI fusion was used for target definition; the gross tumor volume (GTV) encompassed the enhancing tumor with $0 \mathrm{~mm}$ margin added for the clinical target volume (CTV) and a 5mm margin for the planning target volume (PTV). She tolerated treatments well without the need for initiation of decadron. Six weeks post treatment; she experienced increased fatigue, headache and difficulties with short term memory. At twelve weeks, her symptoms were improving but her first post radiation MRI scan demonstrated new fingerlike enhancing projections into the medial temporal lobe with increased mass effect. The neuro-radiologist's interpretation was that of early tumor progression. (Figure 1b).

The patient was clinically stable and close surveillance without decadron was elected. Repeat imaging six weeks later

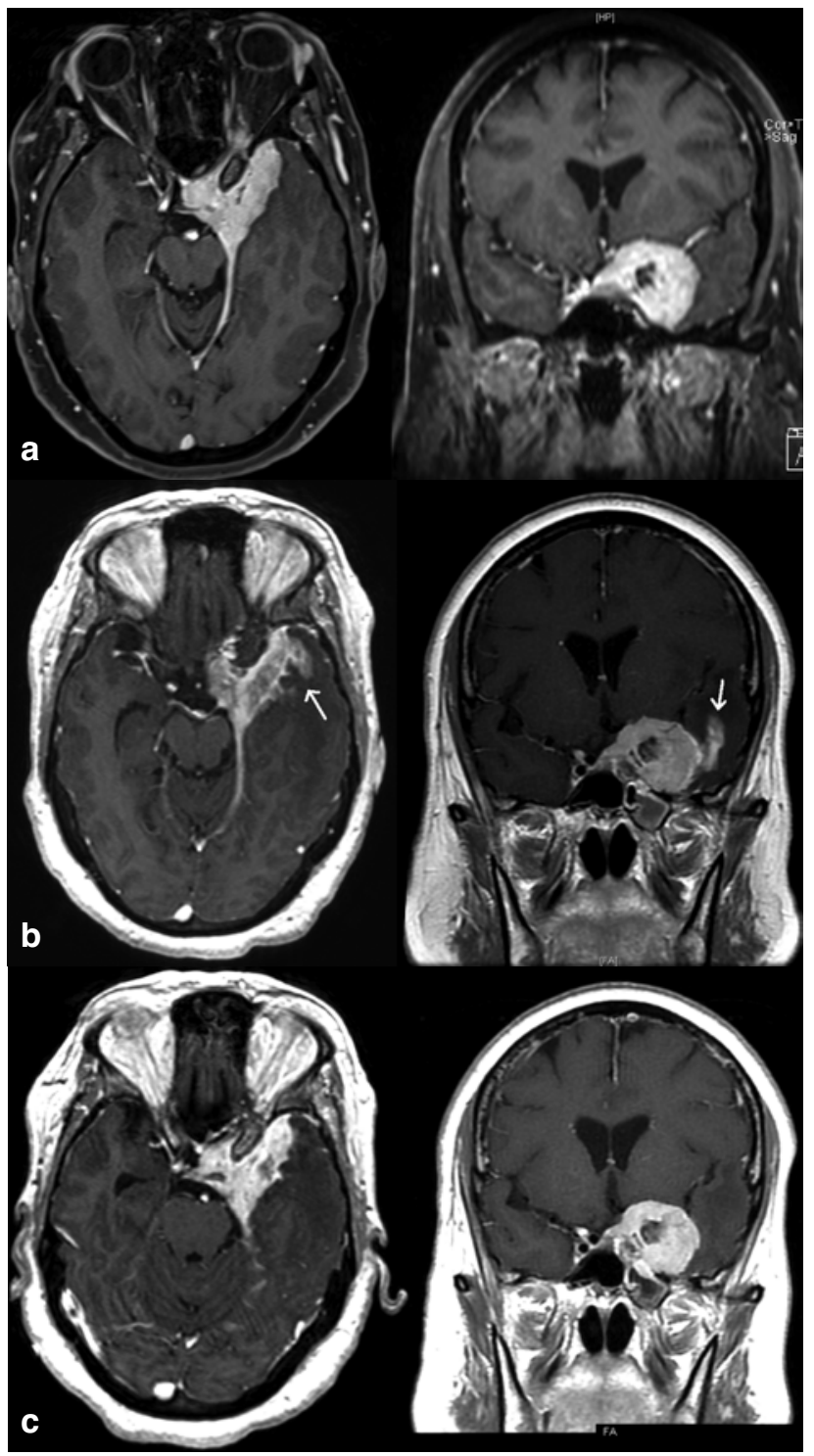

Figure 1: A) T1 contrast enhanced MRI taken at time of diagnosis. B) Twelve weeks post radiation. Arrows represent new areas of enhancement. C) 18 weeks post radiation. This scan shows complete resolution of the new enhancement noted at 12 weeks.

From the Departments of Oncology, University of Western Ontario and London Health Sciences Centre, London, Ontario, Canada.

Received April 7, 2011. Final Revisions Submitted August 12, 2011 Correspondence to: Glenn Bauman, London Regional Cancer Program, 790 Commissioners Rd E, London, Ontario, Canada. 
demonstrated complete resolution of the findings (Figure 1c). At her last follow-up, she was well except for persisting mild memory difficulties. Based on the clinical and radiographic course the changes were attributed to a transient effect of treatment rather than tumor progression was made.

Objective evaluation of tumor response/progression on MRI imaging is a key component of follow up for all primary brain tumor patients. For malignant gliomas, the MacDonald criteria developed in 1990 with a recent update for an objective assessment, are considered robust and have been widely adopted $^{3}$. For meningiomas, there are no tumor specific criteria for assessment of response and is of particular challenge as local control post radiation is usually associated with stable tumor size on serial imaging with radiographic responses observed less frequently and usually over long time frames (years). While new enhancement on imaging after radiation for a primary brain tumor is usually associated with progression treatment effects may confound such assessments. The likelihood of false positives for progression in meningiomas is unknown and in the case of tumors diagnosed on imaging without tissue confirmation, uncertainty regarding histologic grade can confound assessment of response although new imaging techniques may be helpful in this regard..$^{1}$ The early onset of new, transient radiographic enhancement noted in our patient was consistent with radiation related treatment effects; a phenomenon also known as "pseudoprogression".

Most of our understanding and interest about pseudoprogression is derived from the treatments of malignant gliomas. Such post radiation radiographic changes have been described in primary high gliomas as early as $1979 .{ }^{4}$ With contemporary treatment of malignant glioma (typically radiation and concurrent and adjuvant temozolomide), Brandsma et al reported an increase in these false positives studies with radiographic worsening noted to occur in $20-30 \%$ of patients at a median time interval of three months post radiotherapy., The term "pseudoprogression" was introduced in this setting to describe the increase in tumor size or new enhancement within the radiation treatment fields that spontaneously resolve without evidence of further radiological or clinical worsening, allowing for the diagnosis of "pseudoprogression" to be made retrospectively. ${ }^{2}$

Outside of the malignant glioma literature, the evidence is sparse for similar pseudoprogressive states for other primary brain tumors. A literature review (PubMed and Medline) for papers published in English on a pseudoprogression in the setting of meningioma with terms like "pseudoprogression", "early enhancement", "pseudo response" did not reveal any previous documentation of radiographic changes mimicking progression after external beam photon radiotherapy. In meningiomas, the pseudoprogression like changes have been described after boron therapy ${ }^{6}$ and in radiosurgery ${ }^{7}$, however the radiobiological impact of the latter two differs from the fractionated radiation delivery which is typical of external beam radiation. We feel the description of our case to the literature is important as it serves to illustrate the possibility of radiation induced radiographic changes (similar to pseudoprogression) as false positives for progression even in the non-malignant glioma setting. It may be that this is a common but unreported phenomenon in which case this report may serve to stimulate further reports of this phenomenon after conventional, moderated dose fractionated external beam radiation. Timing of imaging may have played a role in the detection of treatment effects in this case; unlike malignant glioma, early (four to six week imaging) may not be routinely performed for benign tumors treated with radiotherapy. Institutional practices that include imaging at longer intervals post treatment (three to six months) may explain the relative paucity of reports of this phenomenon in our literature search, as transient changes at four to six weeks would be missed. A point of interest about our case is that unlike glioblastomas, the treatments of meningiomas have not undergone "intensification" with chemotherapy, a proposed factor for the increase in pseudoprogression or its occurrence earlier. $^{2}$ It would be important to decipher if such a documentation is a result of closer scrutiny with advanced and/or early use of imaging versus novel treatment related or patient factors.

Due to the transient nature, we consider the radiographic changes in our patient as distinct from "radiation necrosis" - a term that has been loosely used in the past to capture all radiographic changes post radiotherapy. True radiation necrosis after radiation alone would be generally considered a late finding (more than six months post treatment) with changes persistent over intervals longer than the six weeks noted in our patient. The brain is a slowly proliferating and late reacting tissue thought to have an $\alpha / \beta$ ratio between $2-2.9$ Gy suggesting a low repair capacity. ${ }^{8}$ There is general consensus that central nervous system necrosis is likely secondary to both neuro-glial damage and secondary vascular changes. Histopathological analyses of these areas show focal coagulative necrosis and demyelination likely secondary to fibrinoid necrosis of small arterial vessels. ${ }^{9}$ Late radiation necrosis is rare below 60 Gy delivered in 1.8-2 Gy per fractions. Dose, fraction size, and treatment volume are considered major risk factors of necrosis, while location, use of chemotherapy, and, diabetes mellitus are less well established risk factors. ${ }^{8}$

The pathophysiology of reversible early radiographic changes after radiation and chemoradiation is poorly understood. Postulated mechanisms include transient endothelial cell damage, leading to disruption of blood brain barrier (BBB), and abnormal vessel permeability causing new or increased contrast enhancement and edema or transient demyelination. Presumably, in some cases, these early radiation induced changes may be more profound leading to subsequent late radiation necrosis. Thus, it is possible that "pseudoprogression" and radiation necrosis represent a continuum of reactive processes to radiation treatments. The inability of standard MRI, to distinguish between BBB disruption secondary to treatment (surgery or radiation), changes in corticosteroid use, tumor progression or late injury effects confounds accurate, early identification of true tumor progression.5 More recently, functional imaging as adjunct to diagnose pseudoprogression has been proposed. Magnetic resonance spectroscopy measuring metabolite concentrations and PET imaging with amino acid tracers can help distinguish between viable and necrotic. ${ }^{5}$ However, even with these advance imaging techniques accurate classification of pseudoprogression versus true tumor progression remains elusive and an area of investigation. ${ }^{1,5}$ 
In summary, our report documents reversible early radiation related imaging changes, distinct from typical radiation necrosis, in a meningioma patient that are analogous to the phenomenon of "pseudoprogression" noted in malignant glioma patients. A limitation of our case report is the absence of tissue confirmation. However, the radiographic appearance was typical of low grade meningiomas, and the stable appearance of the meningioma itself over the time period from pretreatment to post treatment (six months) argues against an atypical or malignant meningioma. This case report serves to remind clinicians of the possibility of pseudoprogression in the setting of brain tumors other than malignant glioma treated with moderate dose, conformal radiotherapy alone. The changes noted in our patient may lie on a spectrum of treatment effects ranging from asymptomatic, reversible imaging changes (pseudoprogression) to frank radiation necrosis and the predictors of evolution of early imaging changes into radiation necrosis requires further study. While dose intensification or combined modality therapy was not a factor in our case (as proposed for the case with pseudoprogression in malignant glioma) other factors such as dose heterogeneity and timing of imaging post radiotherapy may affect the detection of such changes. For example, one might argue for delayed post radiotherapy (four to six month) imaging to avoid "pseudoprogression" false positives due to early post radiation effects. This may not be possible for patients with malignant glioma where adjuvant chemotherapy following radiation is a routine consideration but may be reasonable for patients with extra-axial tumors such as meningioma and schwannomas where adjuvant therapies are not required and early follow-up based on clinical symptoms alone may be reasonable. At the very least, awareness of the possibility of pseudoprogression post radiotherapy as a consequence of radiation alone for tumors other than malignant glioma is important in order to avoid triggering salvage interventions unnecessarily and to better counsel patients during follow-up to avoid unnecessary anxiety.

\section{REFERENCES}

1. Weber DC, Lovblad KO, Rogers L. New pathology classification, imagery techniques and prospective trials for meningiomas: The future looks bright. Curr Opin Neurol. 2010;23:563-70.

2. Brandsma D, Stalpers L, Taal W, et al. Clinical features, mechanisms, and management of pseudoprogression in malignant gliomas. Lancet Oncol. 2008;9:453-61.

3. Wen PY, Macdonald DR, Reardon DA, et al. Updated response assessment criteria for high-grade gliomas: Response assessment in neuro-oncology working group. J Clin Oncol. 2010;28: 1963-72.

4. Hoffman WF, Levin VA, Wilson CB. Evaluation of malignant glioma patients during the postirradiation period. J Neurosurg. 1979;50:624-8.

5. Clarke JL, Chang S. Pseudoprogression and pseudoresponse: Challenges in brain tumor imaging. Curr Neurol Neurosci Rep. 2009;9:241-6.

6. Miyatake S, Kawabata S, Nonoguchi N, et al. Pseudoprogression in boron neutron capture therapy for malignant gliomas and meningiomas. Neuro Oncol. 2009;11:430-6.

7. Korah MP, Nowlan AW, Johnsone PA, et al. Radiation therapy alone for imaging defined meningiomas. Int J Radiat Oncol Biol Phys. 2010; 76:181-6.

8. Lawrence YR, Li XA, el Naqa I, et al. Radiation dose-volume effects in the brain. Int J Radiat Oncol Biol Phys. 2010;76: S20-7.

9. Schultheiss TE, Kun LE, Ang KK, et al. Radiation response of the central nervous system. Int J Radiat Oncol Biol Phys. 1995;31: 1093-112. 